\title{
ARTICLE
}

\section{GnRH impairs diabetic wound healing through enhanced NETosis}

\author{
Yun Sang Lee ${ }^{1}$, Sung Un Kang ${ }^{1}$, Myung-Hoon Lee ${ }^{1,2}$, Haeng-Jun Kim ${ }^{1,2}$, Chang-Hak Han ${ }^{1,2}$, Ho-Ryun Won ${ }^{3}$, Young Uk Park ${ }^{4}$ and \\ Chul-Ho Kim ${ }^{1,2}$
}

\begin{abstract}
It has been reported that neutrophil extracellular traps (NETs) impair wound healing in diabetes and that inhibiting NET generation (NETosis) improves wound healing in diabetic mice. Gonadotropin-releasing hormone (GnRH) agonists are associated with a greater risk of diabetes. However, the role of $\mathrm{GnRH}$ in diabetic wound healing is unclear. We determined whether GnRH-promoted NETosis and induced more severe and delayed diabetic wound healing. A mouse model of diabetes was established using five injections with streptozotocin. Mice with blood glucose levels $>250 \mathrm{mg} / \mathrm{dL}$ were then used in the experiments. GnRH agonist treatment induced delayed wound healing and increased NETosis at the skin wounds of diabetic mice. In contrast, GnRH antagonist treatment inhibited GnRH agonist-induced delayed wound healing. The expression of NETosis markers PAD4 and citrullinated histone H3 were increased in the GnRH-treated diabetic skin wounds in diabetic mice and patients. In vitro experiments also showed that neutrophils expressed a GnRH receptor and that GnRH agonist treatment increased NETosis markers and promoted phorbol myristate acetate (PMA)-induced NETosis in mouse and human neutrophils. Furthermore, GnRH antagonist treatment suppressed the expression of NETosis markers and PMA-induced NETosis, which were increased by GnRH treatment. These results indicated that GnRH-promoted NETosis and that increased NETosis induced delayed wound healing in diabetic skin wounds. Thus, inhibition of $\mathrm{GnRH}$ might be a novel treatment of diabetic foot ulcers.
\end{abstract}

Keywords: Neutrophils; GnRH; Diabetic wound healing; NETosis

Cellular \& Molecular Immunology (2020) 17:856-864; https://doi.org/10.1038/s41423-019-0252-y

\section{INTRODUCTION}

Diabetes affects $\sim 170$ million people worldwide, and the number of patients is expected to double by $2030 .^{1}$ Diabetic foot ulcers (DFUs) are a major morbidity occurring in $15 \%$ of diabetic patients. $^{2}$ Wound healing complications in diabetic patients are complex and mediated by many factors, including impaired angiogenesis, ${ }^{3,4}$ decreased growth factor production, 5,6 reduced cell migration and proliferation, ${ }^{7}$ and uncontrolled expression of metalloproteinases (MMPs)., 8 Because of the complicated mechanisms involved in DFUs, treatments are not effective, and FDA-approved therapies for DFUs are not routinely used even though protocols for standard care are available.

Recently, studies have reported on the formation of neutrophil extracellular traps (NETs), which appear as web-like structures; NET generation (NETosis) is known as a unique type of cell death of neutrophils and is involved in the pathogenesis of impaired wound healing in diabetic patients. ${ }^{10}$ We have previously reported that high glucose levels accelerate phorbol myristate acetate (PMA)-mediated NETosis. We also reported that levels of the PAD4 protein, which is one of the marker for NETosis, were enhanced in neutrophils of diabetic patients and that DNase treatment for digesting NETs improved diabetic wound healing.
In addition, it has been reported that NETosis and pancreatic beta cell destruction are related. ${ }^{11}$ Taken together, these results suggest that NETosis is involved in diabetes and delayed diabetic wound healing.

Gonadotropin-releasing hormone $(\mathrm{GnRH})$ is a peptide hormone. This hormone is secreted from the hypothalamus and is responsible for the release of follicle-stimulating hormone (FSH) and luteinizing hormone (LH) from the anterior pituitary gland. The main functions of $\mathrm{GnRH}$ are therefore related to the reproductive system. However, the $\mathrm{GnRH}$ receptor is expressed in other cells not related to reproductive function, suggesting that $\mathrm{GnRH}$ plays other roles in the body.

Indeed, it has been reported that $\mathrm{GnRH}$ agonists are associated with a greater risk of diabetes. ${ }^{12}$ Neutrophils express the GnRH receptor and participate in diabetes and diabetic wound healing. Therefore, it is important to investigate the possible relationship between $\mathrm{GnRH}$ and diabetic wound healing.

No studies have reported the role of GnRH in diabetic wound healing. In the present study, we showed that a GnRH agonist accelerated NETosis and that treatment with GnRH antagonist inhibited this process. Furthermore, we showed that GnRH agonist-treated mice displayed more delayed wound healing than

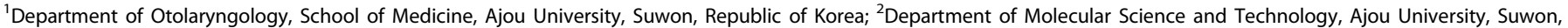

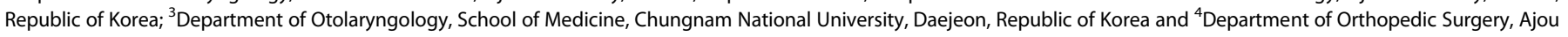
University, Suwon, Republic of Korea

Correspondence: Chul-Ho Kim (ostium@ajou.ac.kr)

These authors contributed equally: Yun Sang Lee, Sung Un Kang

Received: 24 February 2019 Accepted: 2 June 2019

Published online: 19 June 2019 
untreated control mice in a model of diabetes and that $\mathrm{GnRH}$ antagonist treatment inhibited the impairment resulting from $\mathrm{GnRH}$ agonist treatment. These results suggested that $\mathrm{GnRH}$ impairs diabetic wound healing and that inhibition of $\mathrm{GnRH}$ signaling may be a useful strategy for the treatment of diabetic wound healing.

\section{MATERIALS AND METHODS}

Ethics statement

This study was performed according to the Declaration of Helsinki guidelines and was approved by the Ajou University Hospital Institutional Review Board. Informed consent was obtained from the patients.

The protocol for animal use was approved by the Committee for Ethics in Animal Experiments of Ajou University School of Medicine.

Mice

C57BL/6 mice were housed in an environmentally controlled room with a 12-h/12-h light/dark cycle and free access to laboratory chow and water. Mice at 8-12 weeks of age were used. The protocol for animal use was approved by the Ajou University Medical Science Committee.

Cell line and reagents

The HL-60 cell line was purchased from the Cell Line Service (Eppelheim, Germany) and was grown as monolayer cultures in Roswell Park Memorial Institute (RPMI)-1640 medium supplemented with $10 \%$ fetal bovine serum, $1 \%$ penicillin, and $1 \%$ streptomycin. TRIzol reagent was purchased from Invitrogen (Carlsbad, CA).

Induction of diabetes and generation of peripheral wounds in mice

A diabetic mouse model, which is induced with streptozotocin (STZ), was used in the first part of the study to demonstrate that NETosis induces delayed diabetic wound healing in mice, ${ }^{13}$ and this mouse model of diabetes was widely used to study NETosis under diabetic conditions. ${ }^{10}$ Thus, we induced diabetes in mice with STZ. C57BL/6 mice at 8-12 weeks of age were intraperitoneally injected with $40 \mathrm{mg} / \mathrm{kg} \mathrm{STZ}^{14}$ (Sigma-Aldrich, St. Louis, MO, USA) every day for 5 days. Control mice were treated with daily injections of citrate buffer. Serum glucose was measured from mouse tail veins using a glucometer. Once serum glucose levels reached $250 \mathrm{mg} / \mathrm{dL}$, the mice were followed with daily measurements for 1 week prior to use in experiments. Wounds were induced on shaved back skins using 6-mm punch biopsies. Full-thickness skin was removed, exposing the underlying muscle.

Immunohistochemistry for citrullinated histone H3 and PAD4 To compare the expression levels of citrullinated histone $\mathrm{H} 3$ and PAD4 between human skin tissues from control subjects without diabetes and patients with diabetes, we performed immunohistochemical staining as described previously with modifications. ${ }^{15}$ In brief, skin tissues from control subjects without diabetes and patients with diabetic foot ulcers (DFUs) were collected and embedded in paraffin. The paraffin-embedded skin tissue sections were collected on poly-L-lysine-coated slides. After deparaffinization and rehydration, the slides were incubated with 5\% normal goat serum for $1 \mathrm{~h}$ and subsequently incubated with primary antibodies against citrullinated histone H3 (1:200; Cell Signaling Technology, Danvers, MA, USA) and PAD4 (1:50, Cell Signaling Technology) overnight, followed by washing three times with phosphate-buffered saline and staining with secondary antibodies for $2 \mathrm{~h}$ at room temperature. After washing, the slides were stained with a Liquid DAB + Substrate Kit (GBI labs, Mukilteo, WA, USA).
RNA isolation, complementary DNA synthesis, and quantitative real-time polymerase chain reaction (PCR)

Total RNA isolation and first-strand CDNA synthesis were performed as described previously with minor modifications. ${ }^{16}$ Briefly, total RNA was isolated from mouse skin samples or neutrophils using TRIzol reagent (Invitrogen). The first strand of complementary DNA was synthesized from $1 \mu \mathrm{g}$ total RNA using a reverse transcription system (Toyobo, Osaka, Japan). The primer sets for human PAD4, mouse PAD4, human GAPDH, and mouse GAPDH were purchased as QuantiTect primer assays (Qiagen, Hilden, Germany). GAPDH messenger RNA was used as an endogenous control. Real-time PCR was performed using the StepOnePlus real-time PCR system (Thermo Fisher Scientific, Waltham, MA, USA) and a SYBR Green PCR Kit (Toyobo). The amplification program consisted of one cycle at $95^{\circ} \mathrm{C}$ for $10 \mathrm{~min}$, followed by 45 cycles at $95^{\circ} \mathrm{C}$ for $20 \mathrm{~s}, 55^{\circ} \mathrm{C}$ for $20 \mathrm{~s}$, and $72{ }^{\circ} \mathrm{C}$ for $20 \mathrm{~s}$.

\section{Western blotting}

Western blotting was performed as described previously. ${ }^{17}$ Briefly, cells and tissues were collected and lysed with radioimmunoprecipitation assay (RIPA) buffer, containing phosphate and a protease inhibitor cocktail, on ice for $30 \mathrm{~min}$. Following centrifugation at $14,000 \times g$ for $20 \mathrm{~min}$ at $4{ }^{\circ} \mathrm{C}$, proteins in the supernatants were electrophoresed using $10 \%$ polyacrylamide gels and transferred to polyvinylidene fluoride membranes (Pall, NY, USA). The membranes were blocked with $5 \%$ skim milk for $1-2 \mathrm{~h}$ and then incubated with primary antibodies against citrullinated histone H3, histone H3, or GAPDH (Cell Signaling Technology) overnight. The next day, the membranes were washed and incubated with appropriate horseradish peroxidase-conjugated secondary antibodies. Signals were detected using an ECL kit (Amersham, Piscataway, NJ, USA) and visualized by the Las detection program (Fujifilm, Tokyo, Japan).

Mouse and human neutrophil isolation and NETosis assays Peripheral blood neutrophils were isolated using a mouse neutrophil isolation kit as described in the manufacturer's protocols (Miltenyi Biotech, Bergisch Gladbach, Germany). Human neutrophils were isolated from peripheral blood using an EasySep direct human neutrophil isolation kit (Stem Cell Technologies, Vancouver, Canada). Isolated neutrophils were resuspended in RPMI-1640 medium and stimulated with $100 \mathrm{nM}$ PMA. NETosis assays were performed with a NETosis assay kit (Cayman Chemical, Ann Arbor, MI, USA) using the manufacturer's suggested protocols. This study was reviewed and approved by the appropriate Institutional Review Board (AJIRB-BMR-SMP-17-474).

\section{Electron microscopy}

Scanning electron microscopy (SEM) and transmission electron microscopy (TEM) of neutrophils were performed. For SEM, neutrophils were grown on coverslips and fixed with $4 \%$ paraformaldehyde for $1 \mathrm{~h}$ at room temperature. The cells were processed as described previously. ${ }^{18}$ TEM was performed as described previously. ${ }^{19,20}$ In brief, neutrophils were fixed in $2.5 \%$ glutaraldehyde in $0.1 \mathrm{M}$ sodium cacodylate buffer for $1 \mathrm{~h}$ on ice, and the cells were washed and dehydrated through a graded ethanol series. The cells were embedded in Spurr's epoxy resin via propylene oxide and sectioned. Ultrathin sections $(90 \mathrm{~nm})$ were cut on an Ultracut S microtome (Leica, Wetzlar, Germany) and mounted on 200 mesh Athene thin bar grids. The grids were examined using a transmission electron microscope (EM $902 \mathrm{~A}$, Carl Zeiss, Oberkochen, Germany). Representative pictures are shown from at least three independent experiments.

\section{Statistical analysis}

Data are presented as the mean \pm standard error of mean (SEM), and statistical comparisons between groups were performed 
A. Streptozotocin $(40 \mathrm{mg} / \mathrm{kg})$

Everyday for 5 days
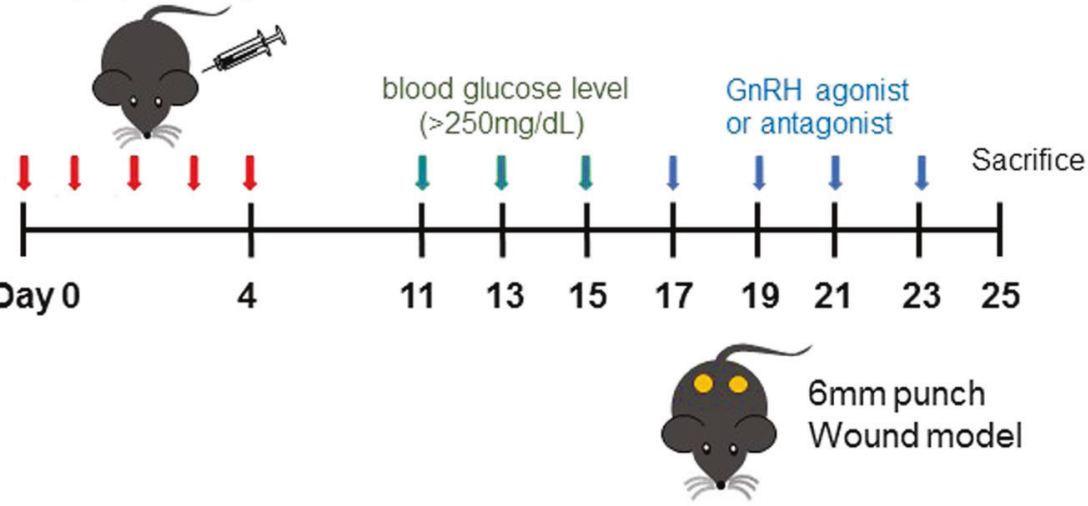

B.

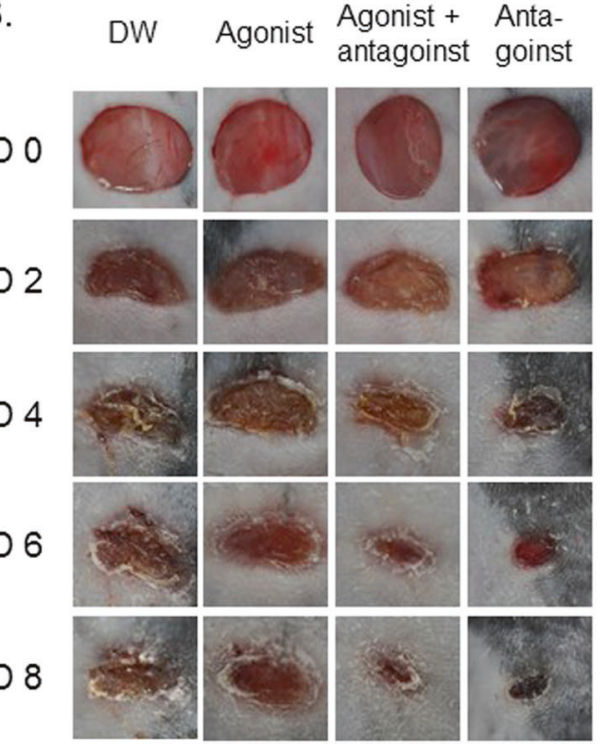

C.
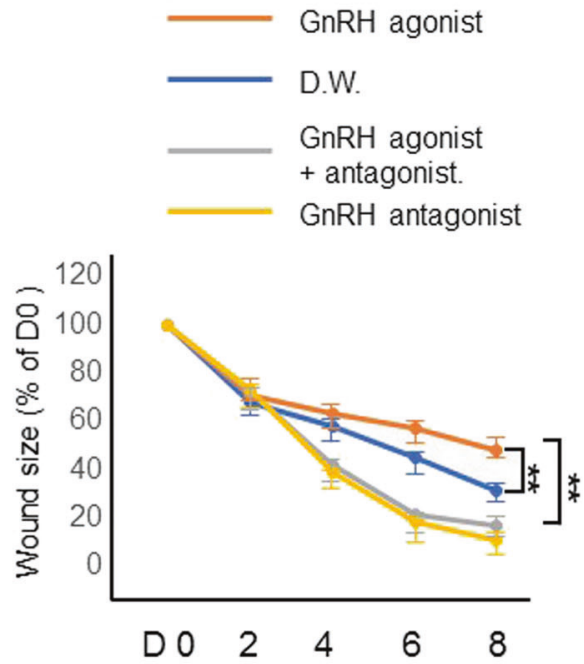

Fig. 1 Gonadotropin-releasing hormone $(\mathrm{GnRH})$ agonist treatment inhibited diabetic wound healing in mice. a The experimental scheme for establishing the mouse model of diabetic wound healing. b Representative photographs of wound healing. $\mathbf{c}$ GnRH agonist treatment inhibited wound healing, and GnRH antagonist treatment promoted this process in a mouse model of diabetic wound healing. ${ }^{* * *} P<0.005$

using unpaired two-sided $t$-tests. All experiments were performed at least three times. Differences with ${ }^{*} P<0.05,{ }^{* *} P<0.005$, and ${ }^{* * *} P<0.005$ were considered statistically significant.

\section{RESULTS}

$\mathrm{GnRH}$ agonist-induced delayed wound healing in the diabetic mouse model

A mouse model of diabetic wound was generated by the intraperitoneal injection of streptozotocin, as described in the Materials and method section; the scheme is illustrated in Fig. 1a. To determine the role of $\mathrm{GnRH}$ in diabetic wound healing, we used a $\mathrm{GnRH}$ agonist and/or $\mathrm{GnRH}$ antagonist to treat the animals every other day. As shown in Fig. 1b, c, GnRH agonist treatment impaired wound healing compared to vehicle treatment. However, GnRH-induced delayed wound healing was improved by treatment with the $\mathrm{GnRH}$ antagonist, suggesting that $\mathrm{GnRH}$ caused delayed wound healing and that treatment with the GnRH antagonist inhibited GnRH-induced impaired wound healing. Notably, GnRH antagonist treatment improved diabetic wound healing compared to no treatment, suggesting that $\mathrm{GnRH}$ antagonist treatment accelerated wound healing during diabetes.
The GnRH agonist increased NETosis in the mouse model of diabetic wound healing

Previous studies have reported that diabetes primes neutrophils to undergo NETosis and that NETosis is involved in impaired wound healing during diabetes. ${ }^{9}$ We therefore determined whether GnRH treatment induced neutrophils to undergo NETosis in the wounds of diabetic mice. It is well known that citrullinated histone $\mathrm{H} 3$ and PAD4 are markers of NETosis. We detected the levels of citrullinated histone H3 and PAD4 in mouse wound tissues using an immunohistochemical assay. Figure $2 \mathrm{a}$, b show that citrullinated histone $\mathrm{H} 3$ levels increased in the GnRH agonisttreated wound samples and that treatment with the $\mathrm{GnRH}$ agonist together with the GnRH antagonist decreased the elevated citrullinated histone $\mathrm{H} 3$ levels. Consistently, PAD4 expression was also increased in $\mathrm{GnRH}$ agonist-treated mouse wounds when compared with that in untreated control and GnRH antagonisttreated wounds. These results suggested that NETosis was involved in the GnRH-induced impairment in wound healing. We also determined the expression levels of citrullinated histone H3, MMP2, and MMP9 using western blotting of mouse wound tissues. Figure $2 \mathrm{a}, \mathrm{b}$ show that citrullinated histone $\mathrm{H} 3$ levels increased in the GnRH-treated wound samples (Fig. 2c). However, 


\section{Control}

\section{$\mathrm{GnRH}$ agonist $\quad \mathrm{GnRH}$ antagonist}

GnRH agonist+ antagonist

A. citrullinated $\mathrm{H} 3$
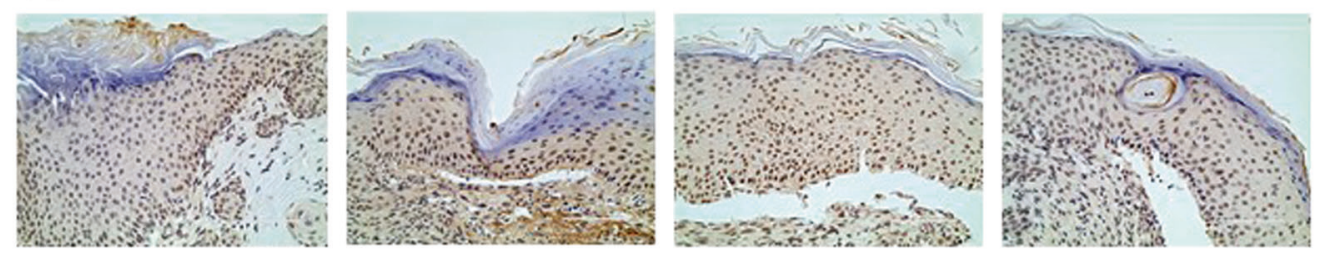

B. PAD4
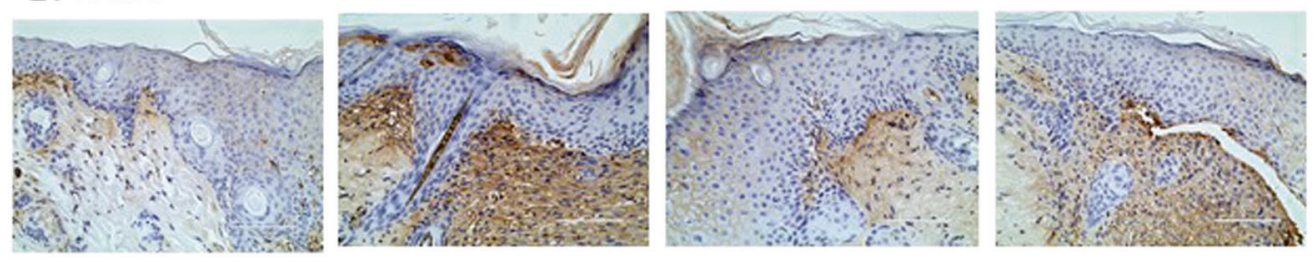

$400 X$

C.

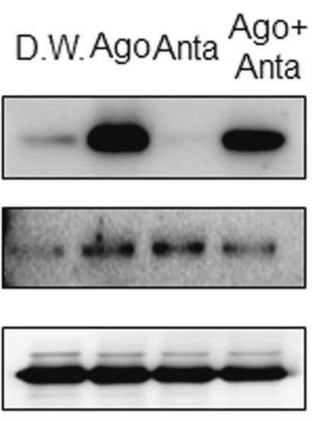

D.

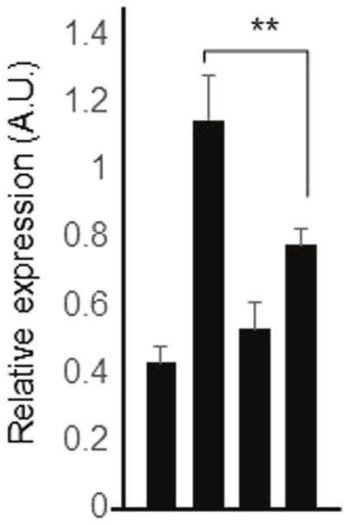

agonist - + - +

\section{Citrullinated} histone $\mathrm{H} 3$

\section{$\mathrm{GnRH}$ Receptor} GAPDH

Fig. 2 Gonadotropin-releasing hormone (GnRH) agonist treatment increased PAD4 expression, a marker of NET generation (NETosis), in a mouse model of diabetic wound healing. Immunohistochemistry for a citrullinated histone H3 and $\mathbf{b}$ PAD4 at wound sites in the mouse model of diabetic wound healing. GnRH treatment increased the expression of citrullinated histone H3 and PAD4, which are markers for NETosis, and $\mathrm{GnRH}$ antagonist treatment suppressed their expression. c Western blotting of citrullinated histone $\mathrm{H} 3$ and $\mathrm{GnRH}$ receptor expression in

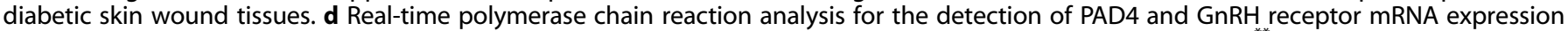
levels in diabetic skin wound tissues. Data are from three independent experiments with four mice per group. ${ }^{* *} P 0.005$

MMP2 and MMP9 expression levels were unchanged in the samples, suggesting that GnRH treatment did not affect MMP expression. We performed real-time PCR analysis to determine the transcript levels in mouse wound tissues. The level of PAD4 was increased in the GnRH-treated samples compared to that in control samples. In addition, GnRH antagonist treatment inhibited GnRH agonist-induced PAD4 expression (Fig. 2d). Together, these results confirmed that GnRH treatment induced NETosis in the skin wounds of diabetic mice.

Expression of NETosis markers and the GnRH receptor increased in human diabetic wounds

We determined the expression levels of citrullinated histone $\mathrm{H} 3$ and PAD4 using an immunohistochemical assay of wound samples from diabetic patients. As previously reported, ${ }^{9}$ citrullinated histone $\mathrm{H} 3$ and PAD4 expression increased in the diabetic wound areas compared with that the normal skin areas (Fig. 3a, b). Real-time PCR analysis also revealed that PAD4 expression increased in the wound areas of diabetic patients when compared to that in the normal skin areas, which confirmed the data in humans and mice with diabetes (Fig. 3d). Notably, western blotting revealed that $\mathrm{GnRH}$ receptor expression in the skin wounds from diabetic patients increased when compared to that in normal skin (Fig. 3c). In addition, we detected the level of the $\mathrm{GnRH}$ receptor in human neutrophils. Figure 3 e shows that the level of $\mathrm{GnRH}$ receptor increased in PMA-stimulated neutrophils, and GnRH agonist-treated PMA-stimulated neutrophils showed increased GnRH receptor expression, and GnRH 
A citrullinated $\mathrm{H} 3$

Normal skin
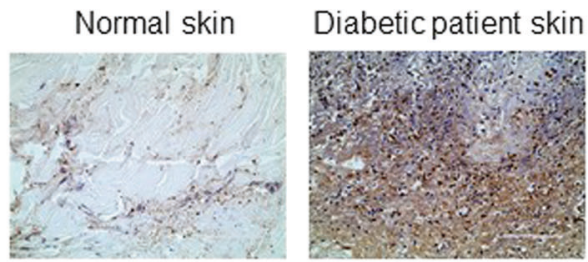

B PAD4
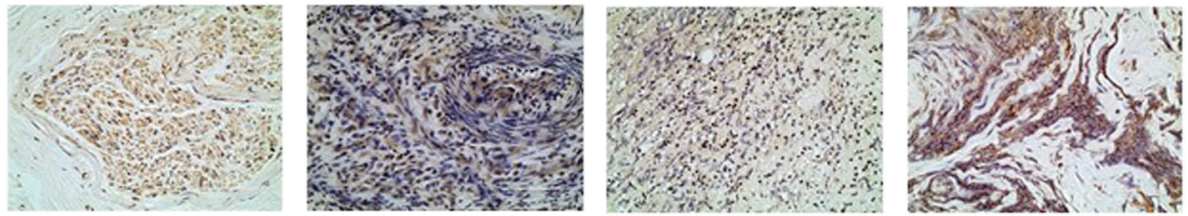

$400 X$
C Patient skin tissue

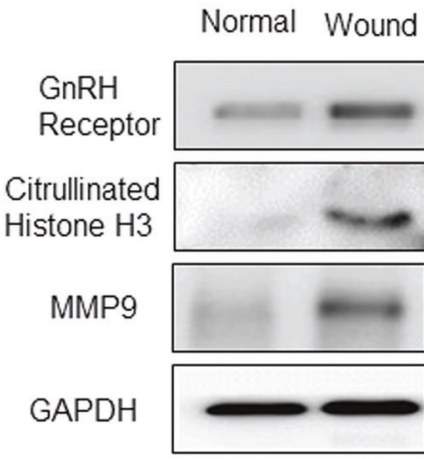

D

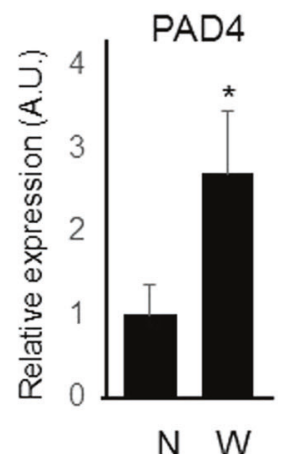

E Patient neutrophils

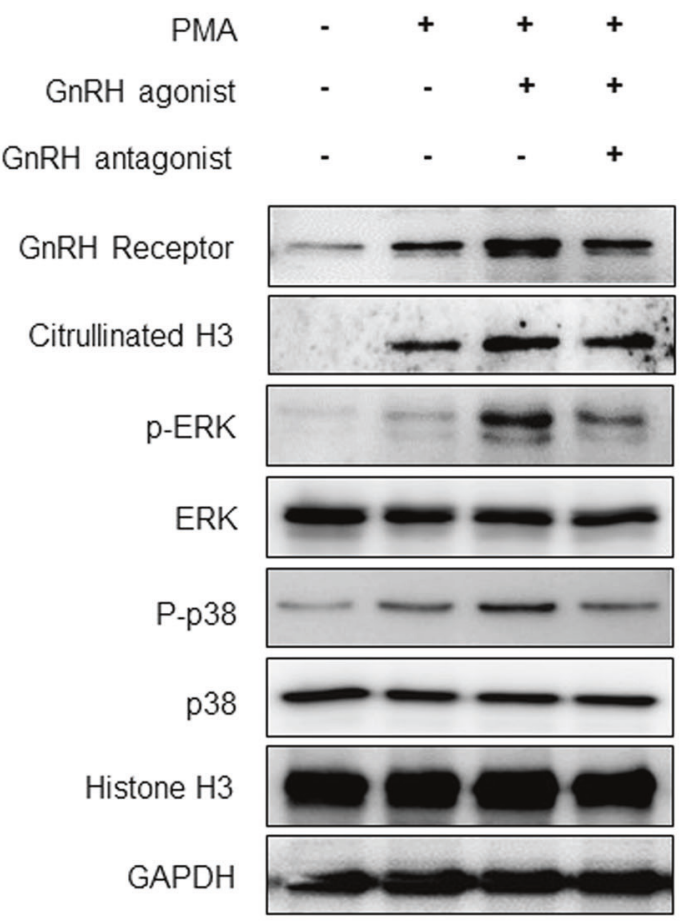

Fig. 3 Expression of citrullinated histone $\mathrm{H} 3$ and PAD4 in diabetic patient skin samples. Immunohistochemistry for a citrullinated histone H3 and b PAD4 showed that citrullinated histone H3 and PAD4 expression was increased in the diabetic wound areas compared with that in normal skin areas. c Western blotting for the expression of the gonadotropin-releasing hormone (GnRH) receptor, citrullinated histone $\mathrm{H} 3$, and MMP9 in normal and diabetic skin wound areas. d Real-time polymerase chain reaction analysis showed that the PAD4 mRNA level was higher in diabetic skin wound areas when compared to that in normal skin areas. e Western blotting using normal human neutrophils. After the neutrophils were isolated from normal human blood, western blotting was performed. The gonadotropin-releasing hormone (GnRH) receptor and citrullinated histone $\mathrm{H} 3$ expression increased after $\mathrm{GnRH}$ agonist treatment, and $\mathrm{GnRH}$ antagonist treatment suppressed this increase in expression. p-ERK and p-P38 levels were detected to confirm the activation of the GnRH signaling pathway. A representative example of three independent experiments is shown. ${ }^{*} P<0.05$

antagonist-treated neutrophils showed decreased GnRH receptor expression compared with $\mathrm{GnRH}$ agonist-treated neutrophils. Citrullinated histone $\mathrm{H} 3$ expression levels were also increased in $\mathrm{PMA} / \mathrm{GnRH}$ agonist-treated neutrophils, suggesting that $\mathrm{GnRH}$ agonist treatment induced $\mathrm{GnRH}$ receptor expression in neutrophils during NETosis.
The GnRH agonist promoted NETosis in mouse and human neutrophils

To determine whether GnRH treatment promoted PMA-induced NETosis, we isolated mouse neutrophils, treated them with PMA/ $\mathrm{GnRH}$ agonist and/or GnRH antagonist, and performed scanning electron microscopy (SEM) and transmission electron microscopy 


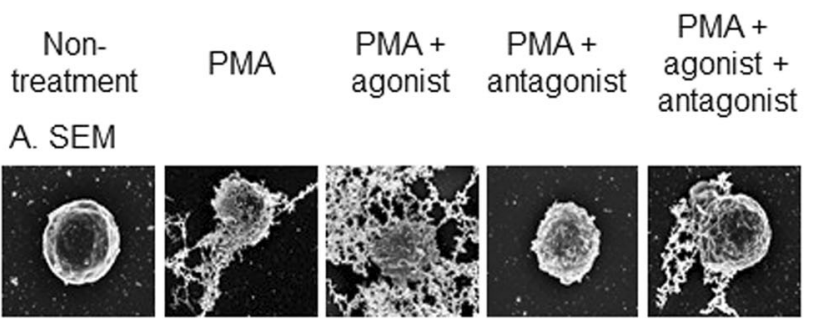

B. TEM

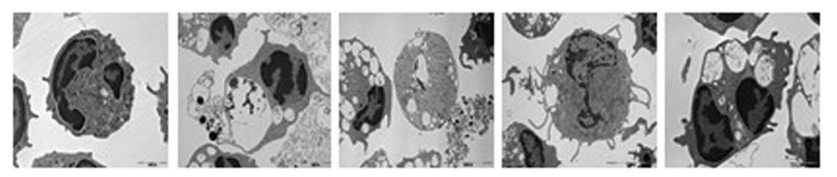

C

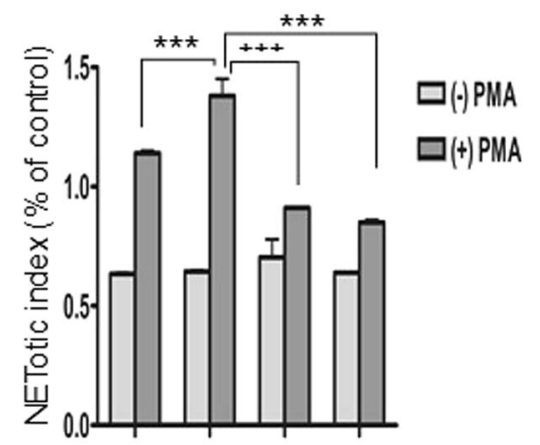

D.

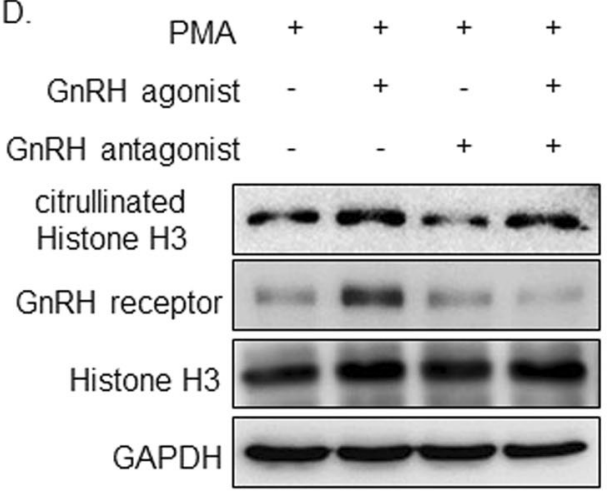

$\mathrm{E}$

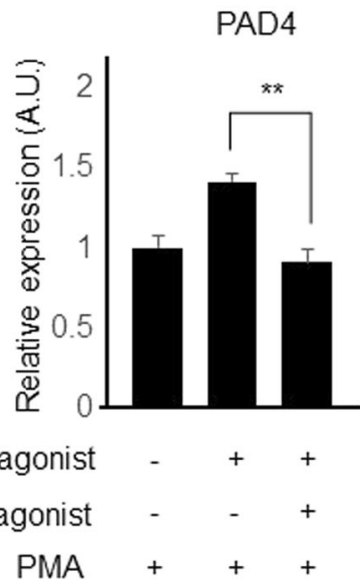

Fig. 4 Gonadotropin-releasing hormone $(\mathrm{GnRH})$ agonist treatment promoted NET generation (NETosis), whereas GnRH antagonist treatment inhibited this process. a Scanning electron microscopy and $\mathbf{b}$ transmission electron microscopy of mouse neutrophils. PMA treatment induced NETosis, and phorbol myristate acetate (PMA)/GnRH agonist treatment induced additional NETosis. GnRH antagonist treatment inhibited increased NETosis. c A NETosis assay showed that GnRH treatment increased NETosis and that GnRH antagonist treatment inhibited the increased NETosis in mouse neutrophils. $\mathbf{d}$ Western blotting of mouse neutrophils. Phorbol myristate acetate (PMA)/GnRH agonist treatment induced more citrullinated histone $\mathrm{H} 3$ and $\mathrm{GnRH}$ receptor expression than treatment with PMA alone. e Real-time polymerase chain reaction experiments showed that GnRH agonist treatment increased PAD4 expression and that GnRH antagonist treatment inhibited PAD4 expression in mouse neutrophils. A representative example of three independent experiments is shown. ${ }^{* *} P<0.005$ and ${ }^{* * * *} P<0.0005$

(TEM) (Fig. 4a, b). Untreated neutrophils displayed a normal phenotype, and PMA-treated neutrophils displayed NETosis phenotypes. Furthermore, PMA/GnRH agonist-treated neutrophils exhibited enhanced NETosis, and PMA/GnRH agonist/GnRH antagonist treatment inhibited GnRH-induced accelerated NETosis. Even treatment with the GnRH antagonist inhibited PMAinduced NETosis. We also performed a NETosis assay to confirm the electron microscopy results. Figure $4 \mathrm{c}$ shows that PMA treatment induced NETosis, and PMA/GnRH agonist treatment induced more NETosis, while PMA/GnRH agonist/GnRH antagonist treatment inhibited PMA/GnRH-induced NETosis, confirming the results of electron microscopy. Western blotting (Fig. 4d) and realtime PCR experiments (Fig. 4e) also showed that GnRH treatment increased citrullinated histone H3 and PAD4 expression in PMAinduced mouse neutrophils during NETosis. These results confirmed that GnRH agonist-induced NETosis and GnRH antagonist inhibited this process in isolated mouse neutrophils. We confirmed that GnRH-induced NETosis in human neutrophils. Consistently, SEM and SYTOX Green staining results showed that $\mathrm{GnRH}$ agonist treatment promoted PMA-induced NETosis and that $\mathrm{GnRH}$ antagonist treatment inhibited PMA/GnRH agonistinduced NETosis (Fig. 5a, b). The NETosis assay of human neutrophils also showed that GnRH agonist treatment accelerated PMA-induced NETosis (Fig. 5c) and increased citrullinated histone H3 levels (Fig. 5d).

Decreased expression of the $\mathrm{GnRH}$ receptor impaired $\mathrm{GnRH}$ promoted NETosis in the HL-60 cell line

To confirm that GnRH-promoted NETosis occurred through GnRH/ $\mathrm{GnRH}$ receptor signaling, we suppressed $\mathrm{GnRH}$ receptor expression with $\mathrm{GnRH}$ receptor-specific siRNAs to determine whether the effect of GnRH on NETosis could be decreased. Transfection of $\mathrm{GnRH}$ receptor-specific siRNAs reduced GnRH receptor expression in the HL-60 cell line (Fig. 6a), showing that siRNAs efficiently inhibited $\mathrm{GnRH}$ receptor expression. Figure $6 \mathrm{~b}$ shows that citrullinated histone $\mathrm{H} 3$ levels did not increase in the $\mathrm{GnRH}$ receptor siRNA-transfected cells, suggesting that the GnRHmediated increase in NETosis occurred through the $\mathrm{GnRH}$ receptor. Consistently, PAD4 expression levels did not increase in GnRH receptor siRNA-transfected cells (Fig. 6c), and the NETosis 
Non-treatment

A. SEM

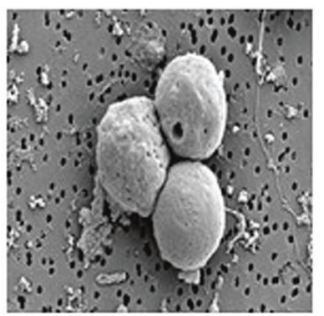

B. Cytox Green
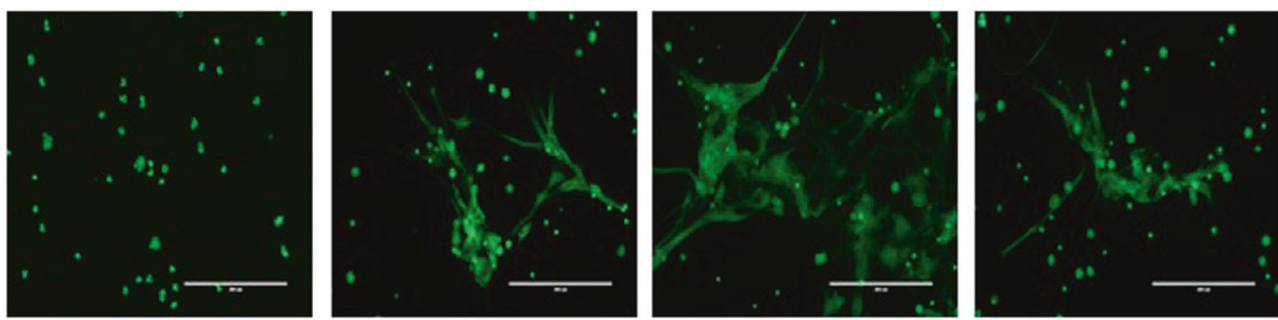

\footnotetext{
C.
}<smiles>CCC[Te]</smiles>

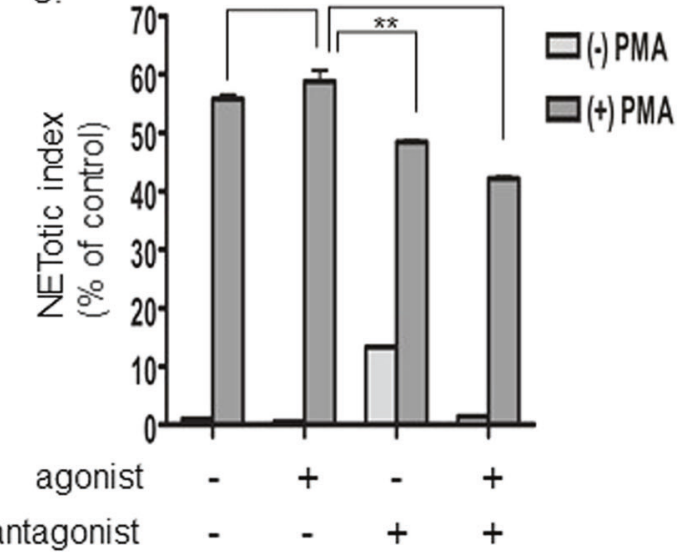

PMA+ GnRH agonist
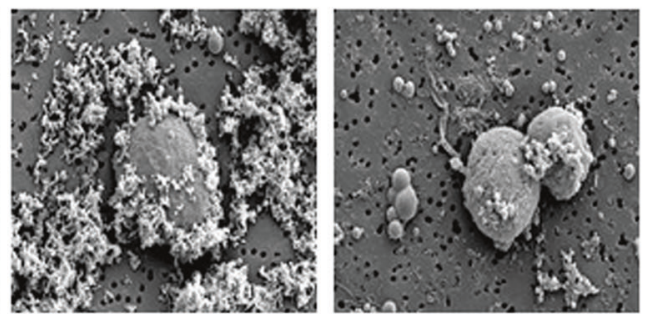

PMA+

$\mathrm{GnRH}$ agonist +

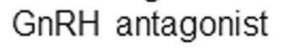

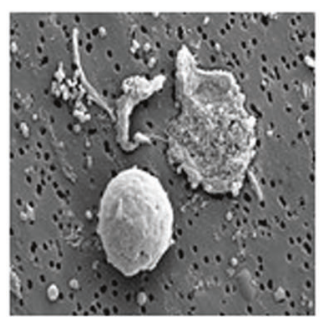

D.

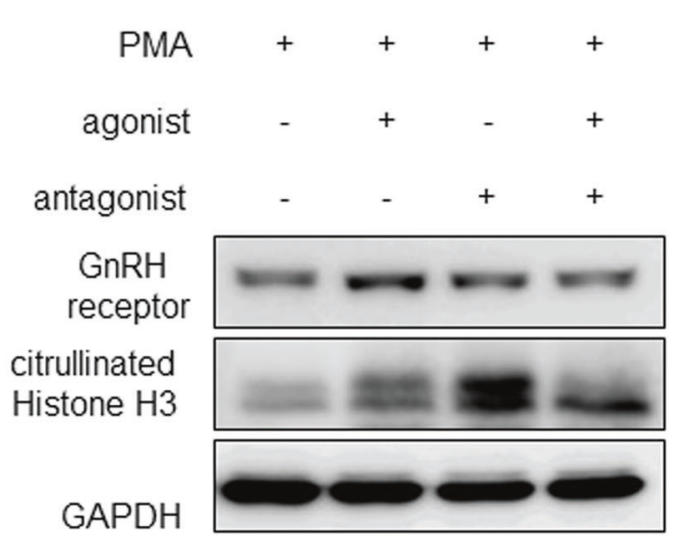

Fig. 5 Gonadotropin-releasing hormone (GnRH) agonist promoted NET generation (NETosis) in human primary neutrophils, whereas GnRH antagonist inhibited this process. a Scanning electron microscopy and b SYTOX Green staining showed that phorbol myristate acetate (PMA)/ GnRH agonist treatment accelerated NETosis and that PMA/GnRH antagonist treatment inhibited the increase in NETosis mediated by the $\mathrm{GnRH}$ agonist. c The NETosis assay showed that GnRH treatment increased NETosis and that GnRH antagonist treatment inhibited the increase in NETosis in isolated human primary neutrophils. d Western blotting of human primary neutrophils. PMA/GnRH agonist treatment induced more citrullinated histone $\mathrm{H} 3$ and $\mathrm{GnRH}$ receptor expression than treatment with PMA alone. GnRH antagonist treatment suppressed the $\mathrm{GnRH}$-mediated induction of expression of the $\mathrm{GnRH}$ receptor and citrullinated histone $\mathrm{H} 3$. A representative example of three independent experiments is shown. ${ }^{* *} P<0.005$

assay also showed that the GnRH receptor was necessary for GnRH-promoted NETosis (Fig. 6d). Taken together, these results indicated that the $\mathrm{GnRH} / \mathrm{GnRH}$ receptor axis was functionally active in neutrophils and that it was involved in the induction of NETosis.

\section{DISCUSSION}

Neutrophils are the most abundant cell type in peripheral blood, and they are the first line of defense against infection. Thus, they play a major role in killing pathogens. In 2004, NETs, weblike structures composed of cytosolic and granule proteins assembled on a scaffold of decondensed chromatin, were discovered $^{21}$ and were subsequently shown to neutralize and kill bacteria, ${ }^{20}$ fungi, $^{21}$ viruses, $^{22}$ and parasites. ${ }^{23}$ However, uncontrolled NETosis can cause immune-related diseases. ${ }^{24}$ Recently, it was suggested that NETosis is involved in delayed wound healing in diabetic patients. ${ }^{10}$ The present study showed that NETosis occurred in the wounds of diabetic patients and that inhibition of NETosis improved impaired wound healing in a mouse model of diabetes.

It was reported that GnRH is associated with a greater risk of diabetes. ${ }^{12}$ However, the roles of $\mathrm{GnRH}$ in impaired wound healing in diabetes and NETosis have not been reported. In the present study, we showed that GnRH promoted increased NETosis, resulting in impaired diabetic wound healing, and that treatment with a GnRH antagonist diminished GnRH-mediated increased NETosis. GnRH does not induce NETosis, but it promotes PMAinduced NETosis, suggesting that GnRH might increase NETosis and exacerbate impaired wound healing during diabetes. 
A

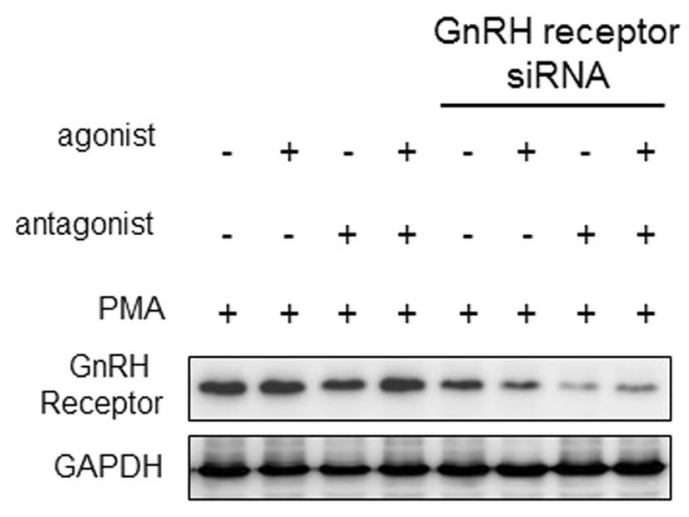

C

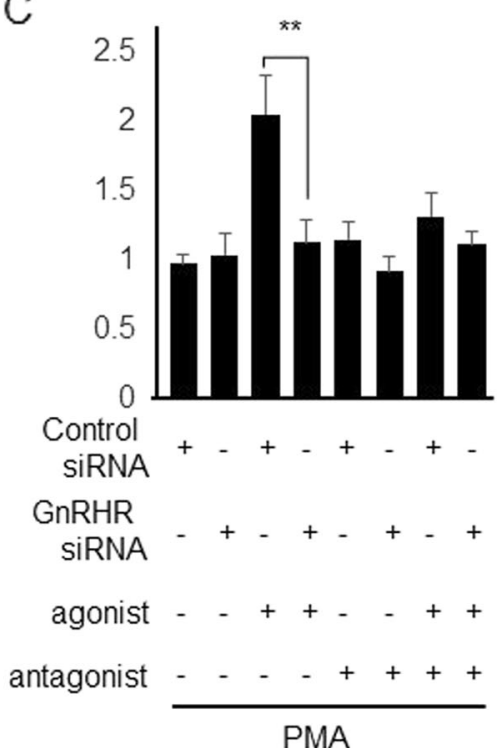

PMA
B
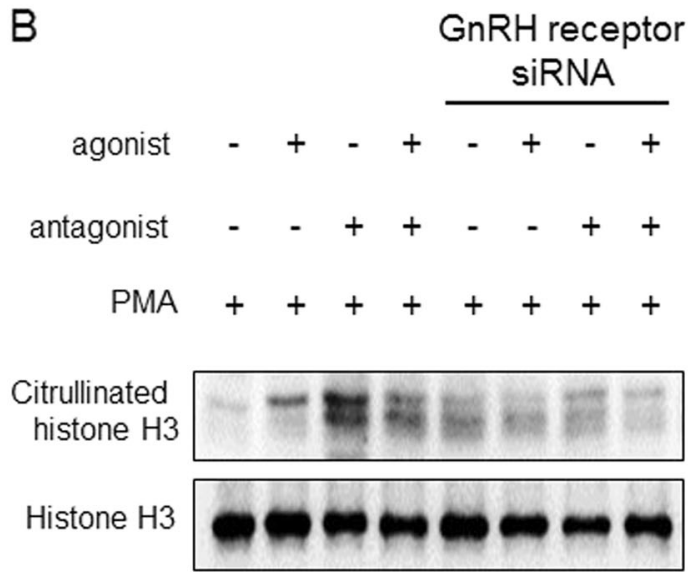

$\mathrm{D}$

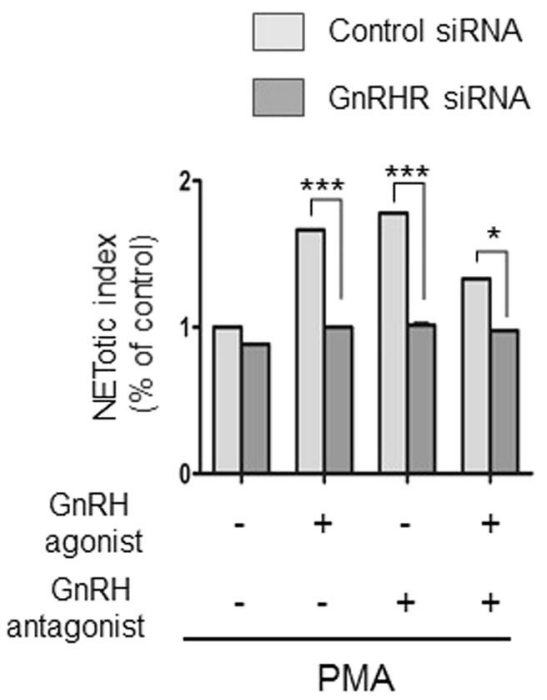

Fig. 6 Decreased expression of the gonadotropin-releasing hormone (GnRH) receptor impaired GnRH-mediated accelerated NET generation (NETosis) in the HL-60 cell line. a Suppression of GnRH receptor expression by GnRH receptor-specific siRNAs inhibited GnRH receptor expression in the HL-60 cell line. b GnRH treatment did not increase the expression of citrullinated histone $\mathrm{H} 3$ in the GnRH receptor siRNAtransfected HL-60 cell line. c Real-time polymerase chain reaction analysis showed that the GnRH receptor siRNA-transfected HL-60 cell line did not have increased PAD4 expression. d The NETosis assay. GnRH agonist treatment did not promote GnRH-mediated increased NETosis in GnRH receptor siRNA-transfected HL-60 cells. A representative example of four independent experiments is shown. ${ }^{*} P<0.05,{ }^{* *} P<0.005$ and ${ }^{* * *} P<0.0005$

$\mathrm{GnRH}$, also known as luteinizing hormone-releasing hormone, is a small peptide hormone of $1.2 \mathrm{kDa}$. The role of $\mathrm{GnRH}$ involves regulation of the neuroendocrine reproductive axis in both males and females. ${ }^{25}$ This neuropeptide is synthesized in hypothalamic neurons and stimulates the synthesis and secretion of gonadotropic hormones such as FSH and $\mathrm{LH}^{26} \mathrm{FSH}$ and $\mathrm{LH}$ are released into the blood circulation and affect gonadal steroidogenesis and gametogenesis. ${ }^{25}$ However, it is also known that GnRH is expressed in several extrahypothalamic cells and tissues. An unexpected finding was that $\mathrm{GnRH}$ is expressed in immune tissues and cells. Analysis of mononuclear cells by real-time PCR analysis revealed that $\mathrm{GnRH}$ genes are expressed in human peripheral blood lymphocytes. ${ }^{27}$ Furthermore, GnRH receptor expression has been reported in immune-related cells and organs. It was shown that the spleen and thymus have GnRH-binding sites; thus, it is assumed that GnRH might contribute to T cell functions. ${ }^{28-31}$

Even though previous reports have shown that immune cells might respond to $\mathrm{GnRH}$ stimulation, we demonstrated for the first time that GnRH stimulated neutrophils to undergo NETosis and that $\mathrm{GnRH}$ antagonist treatment inhibited $\mathrm{GnRH}$-induced NETosis and $\mathrm{GnRH}$-mediated impaired wound healing during diabetes.

NETosis is also involved in many other disease conditions, such as autoimmunity, atherosclerosis, and inflammation. ${ }^{23}$ Thus, a detailed understanding of NETosis and its control might be effective in treating other NETosis-mediated diseases. Although more detailed studies are necessary to elucidate the effects and mechanisms of GnRH on NETosis and impaired diabetic wound healing, our results suggested that treatment with a GnRH 
antagonist may be effective to inhibit GnRH-promoted NETosis and improve impaired wound healing during diabetes.

The advanced development of tissue regeneration and the discovery of new targeting molecules, which are involved in the pathogenesis of diabetic wound healing, and combination therapies might be necessary for treating intractable diabetic wounds. In this study, we demonstrated that GnRH antagonist treatment inhibited NETosis and promoted diabetic wound healing. These results suggest that $\mathrm{GnRH}$ antagonist treatment may be useful for intractable DFUs.

\section{ACKNOWLEDGEMENTS}

This study was supported by the National Research Foundation of Korea grant funded by the Korean government (MSIP) (No. 2011-0030043 (SRC)) and (2017M3A9F7079339) and the Basic Science Research Program through the National Research Foundation of Korea (NRF) funded by the Ministry of Science, ICT, and Future Planning (2018R1A2B3009008).

\section{AUTHOR CONTRIBUTIONS}

Y.S.L. designed and performed mouse experiments, analyzed the results, and wrote the manuscript; S.U.K. designed and performed electron microscopy and siRNA experiments; M.-H.L. performed IHC, western blot analysis, and mouse experiments; H.-J.K. performed IHC; C.-H.K. performed the NETosis assay; H.-R.W. performed neutrophil isolation and induced and analyzed NETosis; Y.U.P. prepared skin samples and analyzed the results; C.-H.K. supervised experiments and wrote the manuscript.

\section{ADDITIONAL INFORMATION}

Competing interests: The authors declare no competing interests.

\section{REFERENCES}

1. Wild, S., Roglic, G., Green, A., Sicree, R. \& King, H. Global prevalence of diabetes: estimates for the year 2000 and projections for 2030. Diabetes Care. 27, 1047-1053 (2004).

2. Ulbrecht, J. S., Cavanagh, P. R. \& Caputo, G. M. Foot problems in diabetes: an overview. Clin. Infect. Dis. 39, S73-S82 (2004).

3. Falanga, V. Wound healing and its impairment in the diabetic foot. Lancet $\mathbf{3 6 6}$, 1736-1743 (2005).

4. Galiano, R. D. et al. Topical vascular endothelial growth factor accelerates diabetic wound healing through increased angiogenesis and by mobilizing and recruiting bone marrow-derived cells. Am. J. Pathol. 164, 1935-1947 (2004).

5. Galkowska, H., Wojewodzka, U. \& Olszewski, W. L. Chemokines, cytokines, and growth factors in keratinocytes and dermal endothelial cells in the margin of chronic diabetic foot ulcers. Wound Repair Regen. 14, 558-565 (2006).

6. Goren, I., Muller, E., Pfeilschifter, J. \& Frank, S. Severely impaired insulin signaling in chronic wounds of diabetic ob/ob mice: a potential role of tumor necrosis factor-alpha. Am. J. Pathol. 168, 765-777 (2006).

7. Gibran, N. S. et al. Diminished neuropeptide levels contribute to the impaired cutaneous healing response associated with diabetes melliuts. J. Surg. Res. 108, 122-128 (2002).

8. Lobmann, R. et al. Expression of matrixmetalloproteinases and their inhibitors in the wounds of diabetic and non-diabetic patients. Diabetologia 45, 1011-1016 (2002).

9. Ayuk S. M., Abrahamse H., Houreld N. N. The role of matrix metalloproteinases in diabetic wound healing in relaton to photobiomodulation. J. Diabetic Res. 2016, 2897656 (2016)
10. Wong, S. L. et al. Diabetes primes neutrophils to undergo NETosis, which impairs wound healing. Nat. Med. 21, 815-819 (2015).

11. Wang, Y. et al. Increased neutrophil elastase and proteinase 3 and augmented NETosis are closely associated with beta-cell autoimmunity in patients with type 1 diabetes. Diabetes 63, 4239-4248 (2014).

12. Keating, N. L., O'Malley, A. J. \& Smith, M. R. Diabetes and cardiovascular disease during androgen deprivation therapy for prostate cancer. J. Clin. Oncol. 24, 4448-4456 (2006).

13. Fadini, G. P. et al. NETosis delays diabetic wound healing in mice and humans. Diabetes 65, 1061-1017 (2016).

14. Park, L. et al. Suppression of accelerated diabetic atherosclerosis by the soluble receptor for advanced glycation endproducts. Nat. Med. 4, 1025-1031 (1998).

15. Kang, S. U. et al. Opposite effects of non-thermal plasma on cell migration and collagen production in keloid and normal fibroblasts. PLOS ONE 12, e0187978 (2017).

16. Lee, Y. S. et al. Non-thermal atmospheric plasma ameliorates imiquimodinduced psoriasis-like skin inflammation in mice through inhibition of immune responses and up-regulation of PD-L1 expression. Sci. Rep. 7, 15564 (2017).

17. Lee, Y. S., Ahjoku, A.-O., Yu, C.-R. \& Egwuagu, C. E. Retinal cells suppress intraocular inflammation (uveitis) through production of interleukin-27 and interleuin-10. Immunology 132, 492-502 (2011).

18. van Buul, J. D. et al. RhoG regulates endothelial apical cup assembly downstream from ICAM1 engagement and is involved in leukocyte trans-endothelial migration. J. Cell Biol. 178, 1279-1293 (2007).

19. Lee, J. S. et al. Epicatechin protects the auditory organ by attenuating cisplatin-induced ototoxicity through inhibition of ERK. Toxicol. Lett. 199, 308-316 (2010).

20. Brinkmann, V. et al. Neutrophil extracellular traps kill bacteria. Science $\mathbf{3 0 3 ,}$ 1532-1535 (2004).

21. Urban, C. F., Reichard, U., Brinkmann, V. \& Zychlinsky, A. Neutrophil extracellular traps capture and kill Candida albicans yeast and hyphal forms. Cell Microbiol. 8, 668-676 (2006)

22. Saitoh, T. et al. Neutrophil extracellular traps mediate a host defense response to human immunodeficiency virus-1. Cell Host Microbe 12, 109-116 (2012).

23. Walker, M. J. et al. DNase Sda1 provides selection pressure for a switch to invasive group A streptococcal infection. Nat. Med. 13, 981-985 (2007).

24. Papayannopoulos, V. Neutrophil extracellular traps in immunity and disease. Nat. Rev. Immunol. 18, 134-147 (2018).

25. Cheung, L. \& Wong, A. S. Gonadotropin-releasing hormone: GnRH receptor signaling in extrapituitary tissues. FEBS J. 275, 5479-5495 (2008).

26. Cheng, C. K. \& Leung, P. C. K. Molecular biology of gonadotropin-releasing hormone $(\mathrm{GnRH})-\mathrm{I}, \mathrm{GnRH}-\mathrm{Il}$ and their receptors in humans. Endocr. Rev. 26, 283-306 (2005).

27. Sasaki, K. \& Norwitz, E. R. Gonadotropin-releasing hormone/gonadotropinreleasing hormone receptor signaling in the placenta. Curr. Opin. Endocrinol. Deabetes Obes. 18, 401-408 (2011).

28. Chen, H. F. et al. Human peripheral blood mononuclear cells express gonadotropin-releasing hormone $(\mathrm{GnRH}), \mathrm{GnRH}$ receptor and interleuin-2 receptor $\mathrm{y}$-chain messenger ribonucleic acids that are regulated by $\mathrm{GnRH}$ in vitro. J. Clin. Endocrinol. Metab. 84, 743-750 (1999).

29. Batticane, N., Morale, M. C., Gallo, F., Farinella, Z. \& Marchetti, B. Luteinizing hormone-releasing hormone signaling at the lymphocyte involves stimulation of interleukin-2 receptor expression. Endocrinology 129, 277-286 (1991).

30. Marchetti, B. et al. Luteinizing hormone-releasing hormone binding sites in the rat thymus: characteristics and biological function. Endocrinology 125, 1025-1036 (1989).

31. Morale, M. C. et al. Blockade of central and peripheral luteinizing hormonereleasing hormone (LHRH) receptors in neonatal rats with a potent LHRHantagonist inhibits the morphofunctional development of the thymus and maturation of the cell-mediated and humoral immune responses. Endocrinology 128, 1073-1085 (1991). 